\title{
Oleuropein prevents the progression of steatohepatitis to hepatic fibrosis induced by a high-fat diet in mice
}

\author{
Sung Woo Kim ${ }^{1}$, Wonhee Hur ${ }^{1}$, Tian $\mathrm{Zhu} \mathrm{Li}^{1}$, Young Ki Lee ${ }^{1}$, Jung Eun Choi ${ }^{1}$, Sung Woo Hong ${ }^{1}$, \\ Kwang-Soo Lyoo ${ }^{1}$, Chan Ran You ${ }^{1}$, Eun Sun Jung ${ }^{2}$, Chan Kun Jung ${ }^{2}$, Taesun Park ${ }^{3}$, Soo-Jong Um ${ }^{4}$ \\ and Seung Kew Yoon ${ }^{1}$
}

\begin{abstract}
Nonalcoholic steatohepatitis (NASH) is characterized by hepatocyte injury and inflammatory cell infiltration, which has been linked to peripheral insulin resistance and increased levels of triglycerides in the liver. The purposes of this study were to establish a mouse model of NASH by feeding mice a $60 \%$ high-fat diet (HFD) and to demonstrate the anti-fibrotic effects of oleuropein, which has been shown to have anti-oxidant and anti-inflammatory properties, in this HFD-induced mouse model of NASH. C57BL/6 mice were divided into three groups: a regular diet group (Chow), a HFD group and an oleuropeinsupplemented HFD group (OSD), which was fed a $0.05 \%$ OSD for 6 months. The effects of oleuropein in this model were evaluated using biochemical, histological and molecular markers. The expression levels of alpha-smooth muscle actin $(\alpha$-SMA)and collagen type I in the HFD and OSD groups were evaluated using real-time PCR and western blotting. The body weight, biochemical marker levels, nonalcoholic fatty liver disease activity score, homeostasis model of assessment-insulin resistance (HOMA-IR) and leptin levels observed in the HFD group at 9 and 12 months were higher than those observed in the Chow group. The HOMA-IR and leptin levels in the OSD group were decreased compared with the HFD group. In addition, $\alpha$-SMA and collagen type I expression were decreased by oleuropein treatment. We established a NASH model induced by HFD and demonstrated that this model exhibits the histopathological features of NASH progressing to fibrosis. Our results suggest that oleuropein may be pharmacologically useful in preventing the progression of steatohepatitis and fibrosis and may be a promising agent for the treatment of NASH in humans.
\end{abstract}

Experimental \& Molecular Medicine (2014) 46, e92; doi:10.1038/emm.2014.10; published online 25 April 2014

Keywords: fibrosis; nonalcoholic steatohepatitis; oleuropein

\section{INTRODUCTION}

Currently, nonalcoholic fatty liver disease (NAFLD) is the most common liver disease, and its prevalence has increased worldwide because of the over-nutrition that is common in the typical contemporary diet and the widespread lack of physical exercise. ${ }^{1,2}$ NAFLD is associated with a wide spectrum of liver pathogenesis, from simple hepatic steatosis to cirrhosis and nonalcoholic steatohepatitis (NASH), and a subset of NAFLD can progress to hepatocellular carcinoma. ${ }^{2,3} \mathrm{NASH}$ is characterized by hepatocyte injury and inflammatory cell infiltration, which has been linked to peripheral insulin resistance and increased triglyceride (TG) concentrations in the liver. ${ }^{4}$

Although the hepatic manifestations of the metabolic syndrome have been investigated extensively, the mechanisms underlying the progression of NASH to cirrhosis or hepatocellular carcinoma in humans are not fully understood. ${ }^{5}$ In addition, the molecular mechanisms associated with the development and effective treatment of NASH have not been extensively studied because of the lack of appropriate animal models reflecting the exact pathophysiological features of human NASH. ${ }^{6,7}$

To date, numerous attempts have been made to generate an animal model of NASH induced by genetic mutations, pharmacological manipulations and/or diet, and the histopathological features and the pathophysiology of NASH have been successfully demonstrated in several mouse and rat models of NASH induced by a high-fat diet (HFD)..$^{7-9}$ However, the extended exposure to HFD (for 6 months)

\footnotetext{
${ }^{1}$ Department of Internal Medicine and Catholic University Liver Research Center, Seoul, Republic of Korea; ${ }^{2}$ Department of Hospital Pathology, College of Medicine, The Catholic University of Korea, Seoul, Republic of Korea; ${ }^{3}$ Department of Food and Nutrition, Yonsei University, Seoul, Republic of Korea and ${ }^{4}$ Department of Bioscience and Biotechnology/Institute of Bioscience, Sejong University, Seoul, Republic of Korea Correspondence: Professor SK Yoon, Department of Internal Medicine, Seoul St Mary's Hospital, 222, Banpo-daero, Seocho-gu, Seoul 137-701, Republic of Korea.

E-mail: yoonsk@catholic.ac.kr

Received 24 May 2013; revised 11 November 2013; accepted 11 November 2013
} 
results in a reduction in the lifespan of the NASH mouse model. Thus, the progression of NASH to mild fibrosis has not been observed. Here, we purposefully induced the progression of NASH to mild fibrosis over 12 months by exposing mice to a HFD.

Therapeutic strategies for NASH patients include lifestyle interventions to promote weight loss, bariatric surgery and pharmacological interventions (for example, insulin-sensitizing drugs, antioxidants and lipid-lowering drugs). ${ }^{10}$ Of these, lifestyle changes are considered to be the most natural method and have been shown to be more effective than several pharmacological interventions. ${ }^{11}$ In addition, several long-term adverse effects may be associated with pharmaceuticals. Thus, we sought to identify natural products that can be used safely for a long term.

Oleuropein, a secoiridoid derived from olives (Olea europaea), can form a part of a healthy diet, and it is a component of several medicines. ${ }^{9}$ The pharmacological properties of oleuropein include anti-oxidant, antiinflammatory, anti-atherogenic, anticancer, anti-microbial, anti-viral and anti-aging effects, and this phenolic compound has been suggested to exert therapeutic effects against coronary heart disease, Parkinson's disease and Alzheimer's disease. ${ }^{8}$ Lipid metabolism in the liver, body fat accumulation and the plasma concentrations of free fatty acids (FFAs), leptin, and TGs were significantly decreased in rats treated with oleuropein. ${ }^{12}$ Recently, we reported that oleuropein exhibits preventative and therapeutic effects in hepatic steatosis. These effects have been associated with lipogenesis (that is, Wnt10b and FGFR1) and pro-inflammatory (TLR2 and TLR4) signaling pathways in a mouse model of NAFLD. ${ }^{13}$

To further investigate the preventative effects of pharmacological treatment with oleuropein against the progression of hepatic fibrosis from steatohepatitis in a HFD-induced mouse model, we hypothesized that oleuropein may also exert preventative or therapeutic effects against the progression of NASH to liver fibrosis. The purposes of this study were thus to establish an experimental model of NASH by feeding mice a HFD and to evaluate the pharmacological effects of oleuropein in this model.

\section{MATERIALS AND METHODS}

\section{Animal care and the establishment of a NASH model}

C57BL/6 mice (38 5-week-old males) were purchased from Orient Bio (Seongnam, Republic of Korea) and were housed in a standard laboratory animal facility. The mice were raised in a temperaturecontrolled room that was maintained on a 12/12-h light/dark cycle. All animal care and experimental protocols were in accordance with the guidelines for the Care and Use of Laboratory Animals from the Research Supporting Center for Medical Science of the Catholic University of Korea.

The 38 mice were divided into groups that received the following diets: regular diet (Chow) that contained $3.8 \mathrm{kcal} \mathrm{g}^{-1}(50.21 \%$ carbohydrate, $20.78 \%$ protein and $4.8 \%$ fat), HFD that contained $5.2 \mathrm{kcal} \mathrm{g}^{-1}$ (26.3\% carbohydrate, $26.2 \%$ protein and $34.9 \%$ fat) and oleuropein-supplemented HFD (OSD), which contained $0.05 \%(\mathrm{w} / \mathrm{w})$ oleuropein (of approximately $80 \%$ purity; Extrasynthese, Genay,
France) in HFD. A subset of the mice from the Chow and HFD groups were killed after 3 months $(n=2$ for Chow/4 for HFD), 6 months ( $n=4$ for Chow/4 for HFD), 9 months $(n=4$ for Chow/4 for HFD) and 12 months ( $n=4$ for Chow/4 for HFD). Next, eight of the NASH mice that were administered HFD for 6 months were further divided into two experimental groups. The first group $(n=4)$ was killed after being treated with OSD for 3 months, and the second group $(n=4)$ was killed after 6 months of OSD treatment. All animals were weighed and anesthetized with ether. Serum was then obtained from the retro-orbital cavity, and mice were killed before liver tissue collection.

\section{Hepatic histology}

Liver tissue samples were fixed in $3.7 \%$ buffered formalin for $24 \mathrm{~h}$ and then embedded in paraffin wax. The samples were cut into $3-\mu \mathrm{m}$ sections and stained with hematoxylin and eosin (H\&E) for evaluations of steatosis, fat droplets and inflammation. Fibrosis was assessed using Masson's trichrome (MT) stain, and TG accumulation in the liver was examined by oil red $\mathrm{O}$ staining. All stained slides were examined by two experienced pathologists who were unaware of sample history, and consensus was reached for every case. Steatosis, hepatocellular ballooning, lobular inflammation and the stages of fibrosis were determined histopathologically and graded as described previously. ${ }^{14}$ The degree of steatosis was graded using the following four-point scale: grade 0 , steatosis involving $<5 \%$ of hepatocytes; grade 1 , steatosis involving up to $33 \%$ of hepatocytes; grade 2, steatosis involving $33-66 \%$ of hepatocytes; and grade 3, steatosis involving $>66 \%$ of hepatocytes. Lobular inflammation was also graded on a four-point scale: grade 0 , no foci; grade 1 , fewer than two foci per $\times 20$ field; grade 2 , two to four foci per $\times 20$ field; and grade 3 , more than four foci per $\times 20$ field. Hepatocyte ballooning was graded on a three-point scale: 0 , none; 1 , a few balloon cells; and 2, many/prominent balloon cells. For the NAFLD activity score (NAS), features of steatosis, lobular inflammation and hepatocyte ballooning were combined, and the range of values were from 0 to 8 . Cases with scores $\geqslant 5.0$ were considered to exhibit NASH, whereas cases with values $\leqslant 2$ were diagnosed as simple steatosis. The stage of fibrosis was evaluated on a seven-point scale: stage 0 , no fibrosis; stage 1a, mild zone 3 perisinusoidal fibrosis; stage $1 \mathrm{~b}$, moderate zone 3 perisinusoidal fibrosis; stage 1c, portal fibrosis only; stage 2, zone 3 perisinusoidal fibrosis and periportal fibrosis; stage 3, bridging fibrosis; and stage 4, cirrhosis. ${ }^{15}$ All MT-stained slides were scanned using Pannoramic Midi (3D Histech Ltd, Budapest, Hungary). To quantitatively evaluate the degree of fibrosis, we prepared a goldstandard data set using 10 randomly selected microscopic fields on whole slide digital images taken by two pathologists. The hepatic veins were excluded from the analysis (Supplementary Figure S1). Using the Pannoramic Viewer (3D Histech Ltd) software, blue-stained areas and non-stained areas from 10 selected fields were determined by color-based thresholding. The percentage of fibrosis was calculated by dividing the blue-stained area by the total area.

\section{Biochemical analyses}

The aspartate aminotransferase (AST), alanine aminotransferase (ALT), total cholesterol (TC) and TG levels in serum samples were measured using an enzymatic-colorimetric method and a BS-400 automatic biochemical analyzer (Mindray, Shenzhen, China), and FFA levels were measured using an RX Daytona kit (Randox, Antrim, UK). The serum levels of insulin and leptin were examined using the LINCOplex mouse serum adipokine kit (LINCO Research, Inc., 
St Charles, MO, USA), and serum glucose levels were measured using the Free Style Lite glucose meter (Abbott, Alameda, CA, USA). Insulin resistance (IR) was calculated using the homeostasis model assessment (HOMA)-IR with the following formula: (fasting serum glucose $\times$ fasting serum insulin)/22.5. ${ }^{16}$

\section{Quantitative real-time PCR}

Total RNA from liver tissues was isolated using the TRIzol reagent (Invitrogen, Carlsbad, CA, USA), and complementary DNA was synthesized using a reverse transcription system (Promega, Madison, WI, USA). Primers used with Universal Probe Library (UPL) probes were designed by Roche Applied Science (Burgess Hill, UK). The gene-specific primer and probe sequences were as follows: collagen type I, forward $5^{\prime}$-CTGCTGGTGAGAGAGGTGAAC- $3^{\prime}$, reverse 5'-ACCAAGGTCTCCAGGAACAC-3', UPL probe no. 79; alphasmooth muscle actin $\left(\alpha\right.$-SMA), forward $5^{\prime}$-TGACGCTGAAGTATCC GATAGA- $3^{\prime}$, reverse $5^{\prime}$-ACCAAGGTCTCCAGGAACAC-3', UPL probe no. 9. $\beta$-Actin was used from the universal primers with a UPL probe (Roche Applied Science, Mannheim, Germany). The collagen type I and $\alpha$-SMA mRNA levels were assessed (with $\beta$-actin as an internal control) in the same tube under the following conditions: pre-incubation at $95^{\circ} \mathrm{C}$ for $10 \mathrm{~min}$, followed by 45 cycles of $95^{\circ} \mathrm{C}$ for $10 \mathrm{~s}, 60^{\circ} \mathrm{C}$ for $30 \mathrm{~s}$ and $72{ }^{\circ} \mathrm{C}$ for $1 \mathrm{~s}$ with fluorescence acquisition in the FAM and yellow 555 channels at the end of each PCR cycle using the multiplex real-time PCR protocol of the LightCycler 480 Probes Master kit (Roche Applied Science, Germany).

\section{Western blot analysis}

Protein extracts were heated at $100{ }^{\circ} \mathrm{C}$ for $5 \mathrm{~min}$ before loading. Samples were then separated on $10 \%$ sodium dodecyl sulfatepolyacrylamide gels, transferred onto nitrocellulose membranes (Schleicher \& Schuell, Dassel, Germany), and blocked for $1 \mathrm{~h}$ at room temperature in $5 \%$ skim milk. The following primary antibodies were used: monoclonal anti- $\beta$-actin (Sigma-Aldrich, St Louis, MO, USA), monoclonal anti- $\alpha$-SMA (Sigma-Aldrich), polyclonal anti-type I collagen (Abcam, Cambridge, MA, USA). Membranes were incubated with these antibodies overnight at $4{ }^{\circ} \mathrm{C}$ and were subsequently incubated with horseradish peroxidase-conjugated secondary antibodies (Amersham Biosciences, Cardiff, UK) for $2 \mathrm{~h}$ at room temperature. Target proteins were detected using an enhanced chemiluminescence system (Amersham Pharmacia Biotech, Uppsala, Sweden) according to the manufacturer's instructions.

\section{Statistical analyses}

The biochemical results, liver index and histological grades are expressed as the means \pm s.d., and significant differences between the groups were assessed using Mann-Whitney $U$-tests and KruskalWallis tests. Real-time PCR assays were performed in triplicate, and groups were compared by one-way analysis of variance. Pearson's correlation coefficient was used to assess the relationships between gene expression and each group. SPSS software (ver. 11.5; SPSS Inc., Chicago, IL, USA) was used for statistical analyses, and $P$-values $<0.05$ were considered to indicate statistical significance.

\section{RESULTS}

\section{Liver index and biochemical values from serum and liver tissues}

TC, TG, FFA, AST and ALT values in serum, and the body weights and liver indices for the Chow and HFD groups were investigated over the course of 12 months (Table 1). HFD was

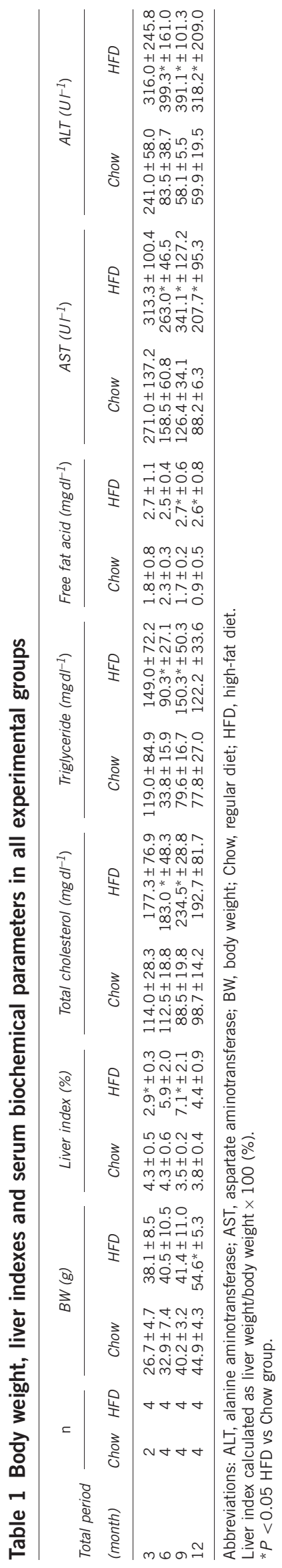


shown to increase body weight compared with the Chow group at all the time points between 3 and 12 months. In addition, the mean body weight of the HFD group at 12 months was significantly higher $(P<0.05)$ than that observed in the Chow group. The liver index (percentage of liver/body weight) was increased in the HFD group compared with the Chow group at the time points between 6 and 12 months. In addition, the mean body weights of the HFD group at 3 and 9 months were significantly higher $(P<0.05)$ than those observed in the Chow group. The serum levels of TC, TG, FFA, AST and ALT in the HFD group were increased compared with the Chow group at 3, 6, 9 and 12 months. The AST and ALT levels in the HFD group at 6,9 and 12 months, the TC and TG levels in the HFD group at 6 and 9 months and the FFA levels in the HFD group at 9 and 12 months were significantly higher $(P<0.05)$ than those observed in the Chow group for each of the respective periods. Morphologically, the HFD group had increased body and liver sizes, and the livers of HFD-fed animals became progressively more yellowish over time (Figure 1A).

\section{Histopathological analysis of NASH}

$\mathrm{H} \& \mathrm{E}$, oil red $\mathrm{O}$ and MT-stained liver tissue sections from the Chow and HFD groups at 3-12 months are shown in Figures 1b-d. No inflammation, steatosis or fibrosis was observed in Chow group livers at 3, 6 or 9 months. However, inflammatory cells and steatosis were observed in the livers of the Chow group at 12 months by H\&E staining (Figures $1 \mathrm{~B}$ and D; a-d). Liver tissue from the HFD group was characterized by hepatic steatosis at 3 to 12 months, and hepatic inflammation (that is, inflammatory cells) was observed using $\mathrm{H} \& \mathrm{E}$ staining at 6-12 months (Figures 1B; e-h). Oil red O staining revealed more lipid droplets in the livers from the HFD group than in those from the Chow group for each time period (Figure 1C), and lipid droplets were rare in Chow group livers at 12 months (Figures $1 \mathrm{C}$; $\mathrm{a}-\mathrm{d}$ ). However, in the livers obtained from the HFD group, increased accumulation of lipid droplets was observed at 3 and 6 months, and this increase was maintained until 12 months (Figure 1C; e-h). Regarding fibrosis, no accumulation of collagen fibers was evident in the Chow group at any time point by MT staining (Figure 1D; a-d). Similarly, the HFD group exhibited no fibrosis at 3 or 6 months, but the HFD group exhibited mild fibrosis at 9 months and then a large increase in fibrosis at 12 months (Figure 1D; e-h).

In this study, we established an animal model of NASH with fibrosis induced by HFD exposure.

\section{NAS scoring in the established NASH mouse model}

The steatosis grade $(0-3)$, NAS score $(0-8)$, fibrosis stage $(0-3)$ and levels of lobular inflammation (0-3) and ballooning (0-2) in the Chow and HFD groups were investigated at 3-12 months (Table 2). The ballooning grade in the HFD group at 3-12 months and the steatosis grade, lobular inflammation grade and NAS score in the HFD group at 6 and 12 months were significantly increased compared with those of the Chow group $(P<0.05)$, as determined by $\mathrm{H} \& \mathrm{E}$ staining. An NAS score $>5$ was considered to indicate NASH. ${ }^{14}$ In our study, we evaluated NASH over 6 months, with an NAS score $>5$ in the HFD group. These results suggest that NASH developed after 6 months in our animal model. The fibrosis grade was determined by evaluating portal fibrosis and interlobular pericellular fibrosis by MT staining. The Chow group had fibrosis stages of $0,1 \mathrm{~A}$ and $\mathrm{IC}$ that were maintained from 3 to 12 months, but the HFD group contained animals at stage 0 (50\%), 1A (25\%) and 1C (25\%) of fibrosis at 3 months; stage $1 \mathrm{~B}(25 \%), 1 \mathrm{C}(50 \%)$ and $2(25 \%)$ fibrosis at 6 months; stage $1 \mathrm{~B}(50 \%)$ and $2(50 \%)$ fibrosis at 9 months; and stage 3 (100\%) fibrosis at 12 months. Thus, mice in the HFD group showed progressively increasing fibrosis in each period.

Thus, collagen accumulation, which suggests the presence of fibrosis, was first observed after 9 months. NAS score evaluations confirmed that HFD led to the development of NASH after 6 months and the presence of fibrosis after 9 months.

\section{Insulin resistance, lipid secretion and fibrosis-related gene expression}

At 3-12 months, HOMA-IR was evaluated to assess the relationship between IR and lipid-related gene-derived leptin secretion in the Chow and HFD groups (Figures 2a and b). Fibrosis-related genes and the collagen type I gene expression levels in the Chow and HFD groups were investigated between 3 and 12 months (Figure 2c). IR in the HFD group was increased compared with the Chow group at each time point, as assessed by HOMA-IR (Figure 2a). IR was increased continuously over time in the HFD group, as assessed by HOMA-IR. Leptin levels in the HFD group during each period were increased when compared with the Chow group (Figure 2b). Leptin levels were elevated between 3 and 9 months in the HFD group. The mRNA expression of collagen type I in the HFD group during each period was increased when compared with the Chow group (Figure 2c). Collagen type I mRNA expression was elevated from 3 to 9 months in the HFD group. However, collagen type I mRNA declined from 9 to 12 months in the HFD group, likely due to the increased injury to hepatocytes observed at 12 months. ${ }^{17}$

These results confirm that IR and lipid accumulation in the HFD-induced mouse model are time dependent, and mRNA expression of type I collagen was first detectable at 6 months.

\section{Liver index and serum biochemistry of oleuropein-treated NASH mice}

The body weights and liver index in NASH mice and the TC, TG, FFA, AST and ALT values in the serum of NASH mice from the HFD and OSD groups were investigated after 3 and 6 months of treatment with oleuropein (Table 3). The body weights observed in the HFD group were increased compared with those in the OSD group during each treatment period. In addition, the mean body weight of the OSD group at 3 months was significantly higher $(P<0.05)$ than that observed in the HFD group. In addition, the liver index in the OSD group (that is, the percentage of liver/body weight) decreased when 
A

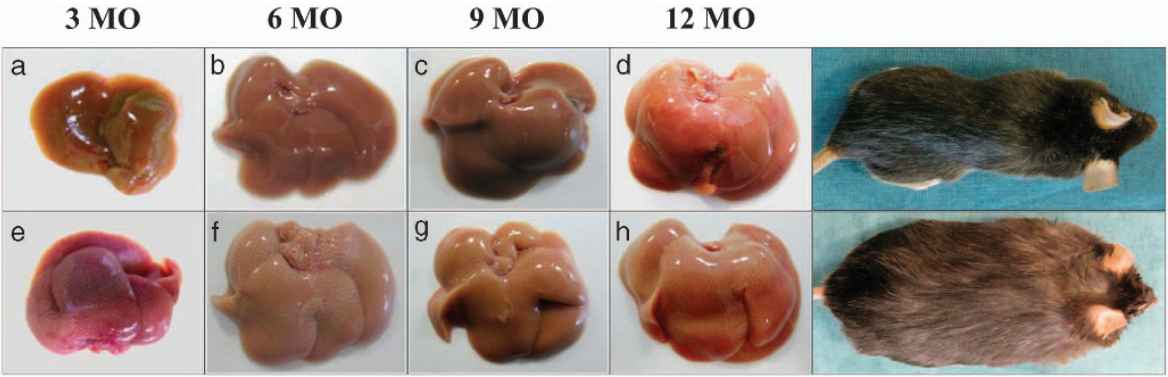

B

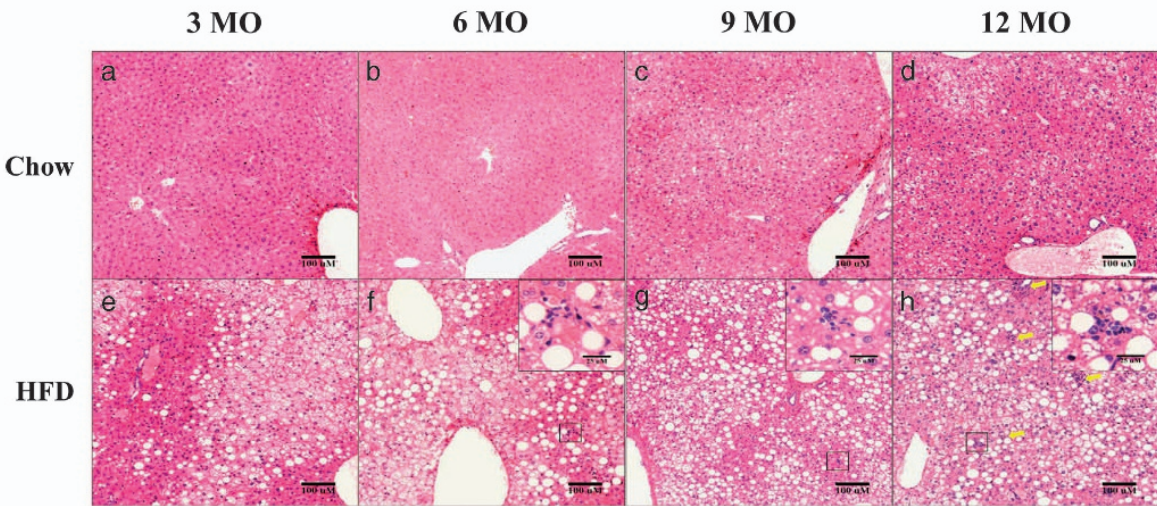

C

3 MO

$6 \mathrm{MO}$

9 MO

12 MO

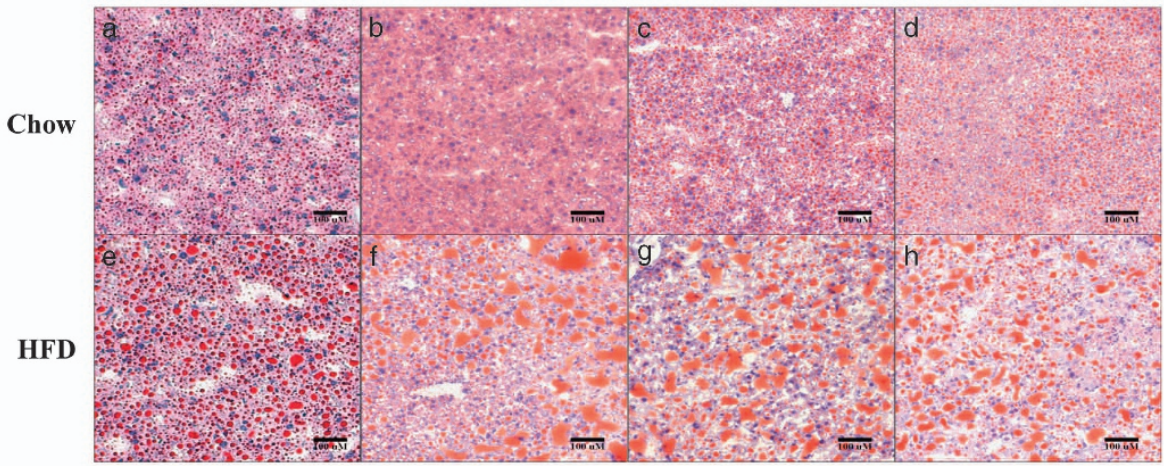

D

3 MO

$6 \mathrm{MO}$

9 MO

12 MO

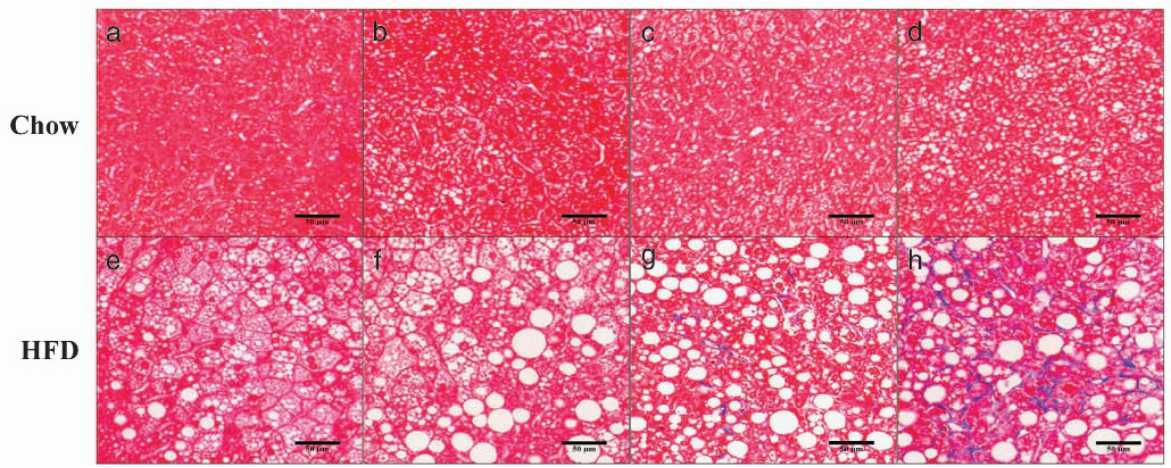

Figure 1 Gross liver morphology, steatosis, and fibrosis in regular diet (Chow)- and high-fat diet (HFD)-fed mice over 12 months. (A) Gross liver and mouse body images; (B) hematoxylin and eosin (H\&E) staining (panels A-H; $\times 150$ object, scale bar $=100 \mu \mathrm{m}$, black box; $\times 400$ object, scale bar $=25 \mu \mathrm{m}$ ); (C) Oil red-O staining (panels A-H; $\times 150$ object, scale bar $=100 \mu \mathrm{m}$ ); (D) Masson's trichrome (MT) staining (panels $A-H ; \times 200$ object, scale bar $=50 \mu \mathrm{m}$, ). H\&E, oil red 0 and MT stains were applied to formalin-fixed paraffin or flash-frozen liver sections. Chow, regular diet-fed mice. HFD, 60\% HFD-fed mice. Chow and HFD mice were fed the respective diets for 3 months (panels a, e), 6 months (panels b, f), 9 months (panels c, g) or 12 months (panels d, h). Inflammatory cells are indicated by yellow arrows. 
Table 2 The NAS between HFD and Chow groups over time

\begin{tabular}{|c|c|c|c|c|c|c|c|c|c|c|c|c|}
\hline \multirow{2}{*}{$\begin{array}{l}\text { Total period } \\
\text { (month) }\end{array}$} & \multirow[b]{2}{*}{ Name } & \multirow[b]{2}{*}{$\mathrm{n}$} & \multirow{2}{*}{$\begin{array}{c}\text { Steatosis grade } \\
(0-3)\end{array}$} & \multirow{2}{*}{$\begin{array}{l}\text { Lobular inflammation } \\
\qquad(0-3)\end{array}$} & \multirow{2}{*}{$\begin{array}{l}\text { Ballooning } \\
\quad(0-2)\end{array}$} & \multirow{2}{*}{$\begin{array}{c}\text { NAS score } \\
(0-8)\end{array}$} & \multicolumn{6}{|c|}{ Fibrosis (n) } \\
\hline & & & & & & & 0 & $1 \mathrm{~A}$ & $1 B$ & $1 C$ & 2 & 3 \\
\hline \multirow[t]{2}{*}{3} & Chow & 2 & $0.0 \pm 0.0$ & $0.0 \pm 0.0$ & $0.0 \pm 0.0$ & $0.0 \pm 0.0$ & 1 & 1 & 0 & 0 & 0 & 0 \\
\hline & HFD & 4 & $1.0 \pm 1.4$ & $0.3 \pm 0.5$ & $0.0 \pm 0.0 *$ & $1.3 \pm 1.9$ & 2 & 1 & 0 & 1 & 0 & 0 \\
\hline \multirow[t]{2}{*}{6} & Chow & 4 & $0.5 \pm 0.6$ & $0.3 \pm 0.5$ & $0.0 \pm 0.0$ & $0.8 \pm 1.0$ & 3 & 0 & 0 & 1 & 0 & 0 \\
\hline & HFD & 4 & $2.5 \pm 0.6^{*}$ & $1.5 \pm 0.6^{*}$ & $1.0 \pm 0.0^{*}$ & $5.0 \pm 0.8^{*}$ & 0 & 0 & 1 & 2 & 1 & 0 \\
\hline \multirow[t]{2}{*}{9} & Chow & 4 & $0.5 \pm 0.6$ & $0.0 \pm 0.0$ & $0.0 \pm 0.0$ & $0.5 \pm 0.6$ & 2 & 2 & 0 & 0 & 0 & 0 \\
\hline & HFD & 4 & $3.0 \pm 0.0 *$ & $1.8 \pm 0.5^{*}$ & $1.8 \pm 0.5^{*}$ & $6.5 \pm 1.0^{*}$ & 0 & 0 & 2 & 0 & 2 & 0 \\
\hline \multirow[t]{2}{*}{12} & Chow & 4 & $0.3 \pm 0.5$ & $0.0 \pm 0.0$ & $0.3 \pm 0.5$ & $1.0 \pm 1.2$ & 2 & 1 & 0 & 1 & 0 & 0 \\
\hline & HFD & 4 & $2.8 \pm 0.5^{*}$ & $2.8 \pm 0.5^{*}$ & $1.8 \pm 0.5^{*}$ & $7.3 \pm 1.0^{*}$ & 0 & 0 & 0 & 0 & 0 & 4 \\
\hline
\end{tabular}

Abbreviations: Chow, regular diet; HFD, high-fat diet; NAFLD, non-alcoholic fatty liver disease; NAS, NAFLD activity score.

${ }^{*} P<0.05$ vs Chow group.
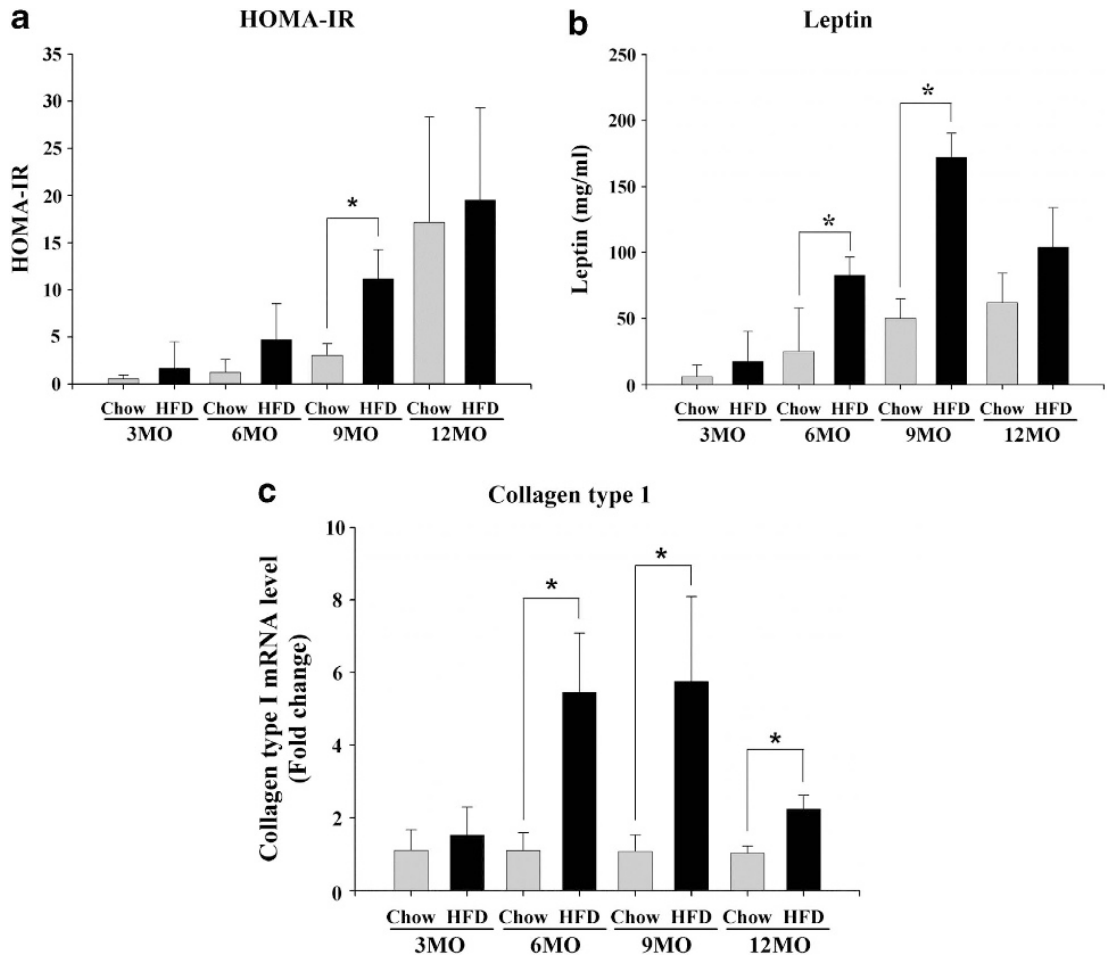

Figure 2 Insulin resistance, lipid levels and fibrosis-related gene expression in the high-fat diet (HFD) and regular diet (Chow) groups over 12 months. Serum (a) homeostasis model of assessment-insulin resistance (HOMA-IR) levels (means \pm s.e.m.); and (b) leptin levels (means \pm s.e.m.). (c) Collagen type I mRNA levels in freshly extracted tissue (means \pm s.e.m.). Chow vs HFD; $n=4, P<0.05$.

compared with the HFD group after 3 months of treatment. Regarding the mean serum TC and TG levels, the OSD group exhibited lower values than the HFD group after both the 3-month and 6-month treatment periods. However, the FFA levels in the OSD group were higher than those observed in the HFD groups after 3 months of treatment, although they were lower in the OSD group after 6 months of treatment. The mean AST and ALT levels in the OSD group were lower than those in the HFD group after a 3-month treatment period. However, the ALT levels decreased, but the AST levels increased after a 6-month treatment period.

\section{Histopathological analysis of liver tissues from} oleuropein-treated NASH mice

MT-stained sections of liver tissues from the HFD and OSD groups after 3 and 6 months of treatment are shown in Figure 3, which presents the average of 10 randomly selected fields. With H\&E staining, it was difficult to distinguish reduced steatosis in the OSD group compared with the HFD group after 3 or 6 months of treatment, but the number of inflammatory cells decreased in response to 3 to 6 months of oleuropein administration. The fibrotic score decreased approximately $47 \%$ in the OSD group compared with the 
HFD group at the 3-month time point, and it significantly decreased approximately $65 \%$ in the OSD group after 6 months $(P<0.05)$. NAS scores were also evaluated using histology (Table 4). The OSD group exhibited only minimal reductions in steatosis grade and ballooning grade after 3 months of oleuropein treatment when compared with the HFD group, but further reductions were observed after 6 months of treatment. However, the OSD group exhibited a decrease in the lobular inflammation score from 1.8 to 0.5 compared with the HFD group after 3 months of treatment $(P<0.05)$. After 6 months of treatment, the lobular inflammation score decreased markedly from 2.8 to 1.0 in the HFD group $(P<0.05)$. Finally, the OSD group was positive for NASH in terms of the NAS score (NAS score $>5$ ), but this score significantly decreased to 4.5 after 3 months of treatment and to 4.3 after 6 months of treatment $(P<0.05)$. With regard to MT staining, the OSD group showed markedly decreased staining after 3 and 6 months. We also evaluated the stage of fibrosis and found that the fibrosis score decreased in the OSD group (1B (25\%) and $1 \mathrm{~A}(75 \%))$ compared with the HFD group $(2(50 \%)$ and $1 \mathrm{~B}(50 \%))$ after 3 months of treatment. This score continued to decrease markedly after 6 months of treatment, at which time the HFD group had significantly higher scores $(3(100 \%))$ than the OSD group $(2(50 \%)$ and $1 \mathrm{C}(50 \%))$.

These results confirm that liver fibrosis and inflammation in the OSD group decreased markedly although the steatosis was not completely healed.

\section{Insulin resistance and leptin secretion in the livers of oleuropein-treated NASH mice}

We evaluated HOMA-IR and leptin levels in the HFD and OSD groups to investigate the relationship between IR and lipid-related gene-derived leptin secretion after 3 and 6 months of treatment (Figures 4a and d). IR in the OSD group was significantly $(P<0.05)$ decreased to approximately $66 \%$ of the level observed in the HFD group after 3 months of treatment, as assessed by HOMA-IR (Figure 4a). IR in the OSD decreased further to approximately $30 \%$ of that in the HFD group after 6 months of treatment (Figure 4c). Leptin secretion in the OSD group was decreased only slightly compared with the HFD group after 3 and 6 months of treatment (Figures $4 \mathrm{~b}$ and $\mathrm{d}$ ).

These data demonstrate that oleuropein reduces serum leptin levels only after 3 months but that it decreases IR at both 3 and 6 months. Accordingly, oleuropein may prevent the progression of NASH to fibrosis by decreasing IR.

\section{Fibrosis-related gene expression in liver tissues from} oleuropein-treated NASH mice

To investigate the expression levels of fibrosis-related genes, the mRNA and protein levels of collagen type I and $\alpha$-SMA were measured after 3 and 6 months of oleuropein treatment (Figure 5). After 3 and 6 months of treatment, both the mRNA and protein levels of $\alpha$-SMA in the OSD group were 

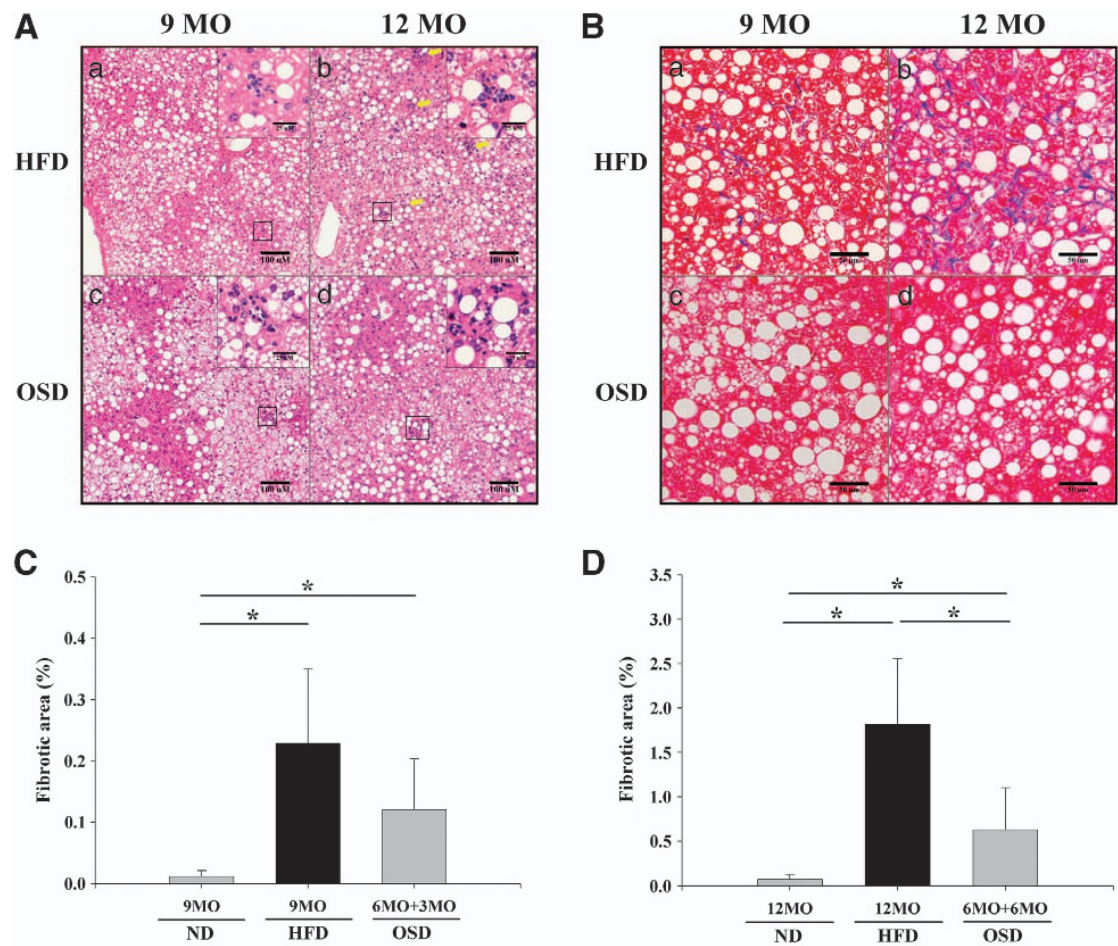

Figure 3 Histological images of livers from an oleuropein-supplemented HFD (OSD) and high-fat diet (HFD) groups after hematoxylin and eosin (H\&E) and Masson's trichrome (MT) staining. (A) H\&E staining (panels A-D; $\times 150$ object, bar $=100 \mu \mathrm{m}$, black box; $\times 400$ object, scale bar $=25 \mu \mathrm{m}$ ); (B) MT staining (panels A-D; $\times 200$ object, scale bar $=50 \mu \mathrm{m}$ ). H\&E and MT stains were applied to formalin-fixed paraffin sections. HFD, 60\% HFD fed mice. OSD, 0.05\% oleuropein mixed with 60\% HFD mice. HFD mice were fed the HFD for 9 or 12 months (panels a, b). The OSD mice were fed oleuropein in conjunction with a HFD after 6 months of the HFD alone (panels c, d). Inflammatory cells are indicated by yellow arrows. (C) The percentage of fibrosis in the OSD group after 3 months of oleuropein treatment $\left({ }^{*} P<0.05\right)$. (D) The percentage of fibrosis in the OSD group after 6 months of treatment $\left({ }^{*} P<0.05\right)$.

Table 4 The NAS between HFD and OSD groups over time

\begin{tabular}{|c|c|c|c|c|c|c|c|c|c|c|c|c|c|}
\hline \multirow{2}{*}{$\begin{array}{l}\text { Total } \\
\text { period } \\
\text { (month) }\end{array}$} & \multirow{2}{*}{$\begin{array}{l}\text { Treatment } \\
\text { period } \\
\text { (month) }\end{array}$} & \multirow[b]{2}{*}{ Name } & \multirow[b]{2}{*}{$\mathrm{n}$} & \multirow{2}{*}{$\begin{array}{l}\text { Steatosis } \\
\text { grade } \\
(0-3)\end{array}$} & \multirow{2}{*}{$\begin{array}{c}\text { Lobular } \\
\text { inflammation } \\
(0-3)\end{array}$} & \multirow[b]{2}{*}{$\begin{array}{l}\text { Ballooning } \\
\quad(0-2)\end{array}$} & \multirow[b]{2}{*}{$\begin{array}{c}\text { NAS score } \\
(0-8)\end{array}$} & \multicolumn{6}{|c|}{ Fibrosis (n) } \\
\hline & & & & & & & & 0 & $1 A$ & $1 B$ & $1 C$ & 2 & 3 \\
\hline 9 & 3 & HFD & 4 & $3.0 \pm 0.0$ & $1.8 \pm 0.5$ & $1.8 \pm 0.5$ & $6.5 \pm 1.0$ & 0 & 0 & 2 & 0 & 2 & 0 \\
\hline & & OSD & 4 & $2.8 \pm 0.5$ & $0.5 \pm 0.6^{*}$ & $1.3 \pm 0.5$ & $4.5 \pm 0.6^{*}$ & 0 & 3 & 1 & 0 & 0 & 0 \\
\hline 12 & 6 & HFD & 4 & $2.8 \pm 0.5$ & $2.8 \pm 0.5$ & $1.8 \pm 0.5$ & $7.3 \pm 1.0$ & 0 & 0 & 0 & 0 & 0 & 4 \\
\hline
\end{tabular}

Abbreviations: HFD, high-fat diet; OSD, oleuropein-supplemented HFD group; NAFLD, non-alcoholic fatty liver disease; NAS, NAFLD activity score.

$* P<0.05$ vs HFD group.

lower than in the HFD group. The expression levels of $\alpha$-SMA protein in the OSD group after 3 months of treatment were especially affected by oleuropein treatment and were significantly decreased by approximately $40 \%$ in comparison with the HFD group (Figures 5a, c, e and f). The levels of collagen type I mRNA and protein were slightly reduced in the OSD group compared with the HFD group after 3 and 6 months of treatment (Figures 5b, d, e and f).

These results indicate that oleuropein prevents the progression of liver fibrosis in a mouse model of NASH induced by HFD.

\section{DISCUSSION}

The establishment of suitable animal models is a promising strategy to investigate the pathogenic pathways that interact to contribute to human diseases, and these animal models can facilitate the development of therapeutic agents. However, the validity of most current animal models of NASH, which are induced by various dietary or genetic modifications, is limited by their failure to produce significant steatohepatitis and other pathophysiological features associated with NASH. ${ }^{18,19}$ Our group has previously reported the preventative effect of oleuropein in hepatic steatosis. ${ }^{13}$ In this study, we further 
a

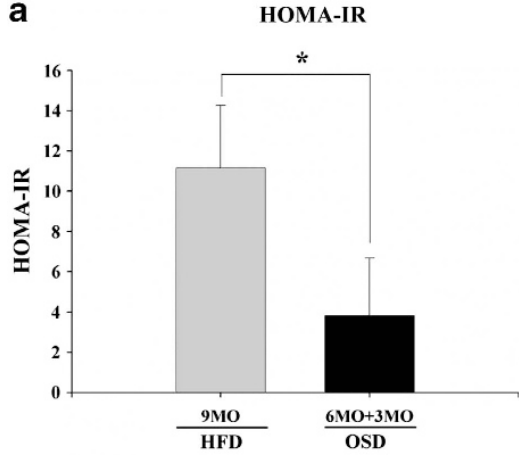

C

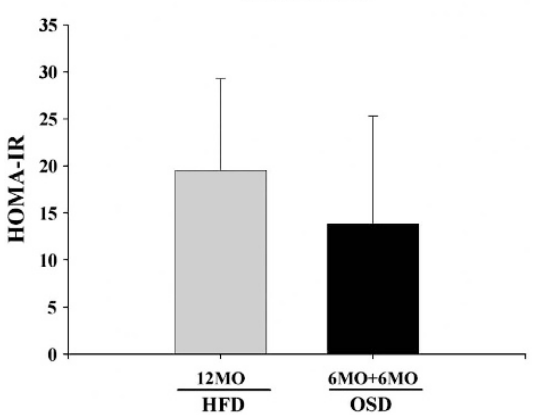

b

Leptin

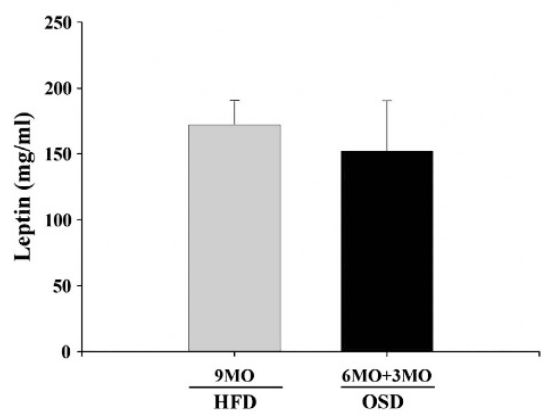

d

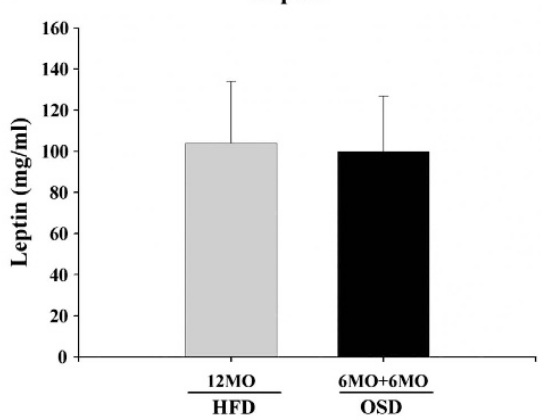

Figure 4 Insulin resistance and lipid levels in the high-fat diet (HFD) and oleuropein-supplemented HFD (OSD) groups after 3 or 6 months of oleuropein treatment in conjunction with a HFD after 6 months of a HFD. Serum (a) homeostasis model of assessmentinsulin resistance (HOMA-IR) levels in OSD mice after 3 months (means \pm s.e.m.); (b) leptin levels in OSD mice after 3 months (means \pm s.e.m.); (c) HOMA-IR levels in OSD mice after 6 months (means \pm s.e.m.); and (d) leptin levels in OSD mice after 6 months (means \pm s.e.m.). HFD vs $0 S D ; n=4, P<0.05$

explored the preventative effects of oleuropein in HFD-treated mice, in which NASH had progressed to liver fibrosis.

It has been recently suggested that animals fed a HFD represent an appropriate model of NASH and that the longterm exposure of rodents to HFD results in the development of many pathogenic features associated with human NASH, including steatosis, inflammation and liver fibrosis. ${ }^{6}$ A murine NASH model administered HFD (where $60 \%$ of all calories are from fat) for 50 weeks exhibited clinical aspects and histopathological changes that were characteristic of human NASH. ${ }^{20}$ In rats, HFD feeding for 48 weeks has been reported to induce pathological features mimicking nearly all of those common in human NASH patients. ${ }^{21}$ Several mouse models of NASH have been induced by diet (HFD or methionine/ choline-deficient diet or a high-sucrose/fructose diet) in either genetically mutated or C57BL/6J mice. ${ }^{7}$ However, these prior mouse models of NASH have been able to model only inflammation, steatosis and IR, not the natural progression of NASH to fibrosis, which is an important feature that should also be investigated. Thus, we attempted to combine aspects of several existing NASH mouse models to develop a new model that mimics the progression of NASH to fibrosis that is observed in humans.

In this study, C57BL/6 mice were fed a $60 \%$ fat diet for 12 months. The HFD group exhibited morbid obesity, increased liver damage and collagen accumulation over time. We also observed increased insulin secretion and hepatic fat accumulation in the HFD group when compared with the Chow group, which has been reported previously. ${ }^{20}$ In humans, IR is an important feature of NASH pathogenesis, and hyperleptinemia can promote hepatic steatosis and steatohepatitis due to changes in insulin secretion and/or the accumulation of hepatic fat. ${ }^{22,23}$ On the basis of our results, we established a NASH mouse model that exhibits pathological features that accurately model this disease in humans. We then investigated the progression of NASH to fibrosis by means of NAS scoring and measuring the mRNA levels of relevant genes. The levels of lobular inflammation and fibrosis in the HFD group were found to be significantly higher than in the Chow group. The increased stages of fibrosis observed in the HFD group were confirmed by the increased levels of collagen type I and $\alpha$-SMA identified by real-time PCR. Indeed, significant levels of perisinusoidal fibrosis and steatohepatitis lesions were observed in mice from the HFD group, but not the Chow group. Thus, mice administered a HFD exhibited many of the changes observed in human NASH progressing to fibrosis.

These features reveal that the pathological severity of NASH was achieved in HFD-fed mice, indicating that the present model may accurately represent a model of the progression of NASH to fibrosis in humans.

To date, only limited data have been available regarding the effects of oleuropein, especially on the liver. However, nearly all of the published studies have reported beneficial effects of oleuropein, provided that it was administered to the NASH 

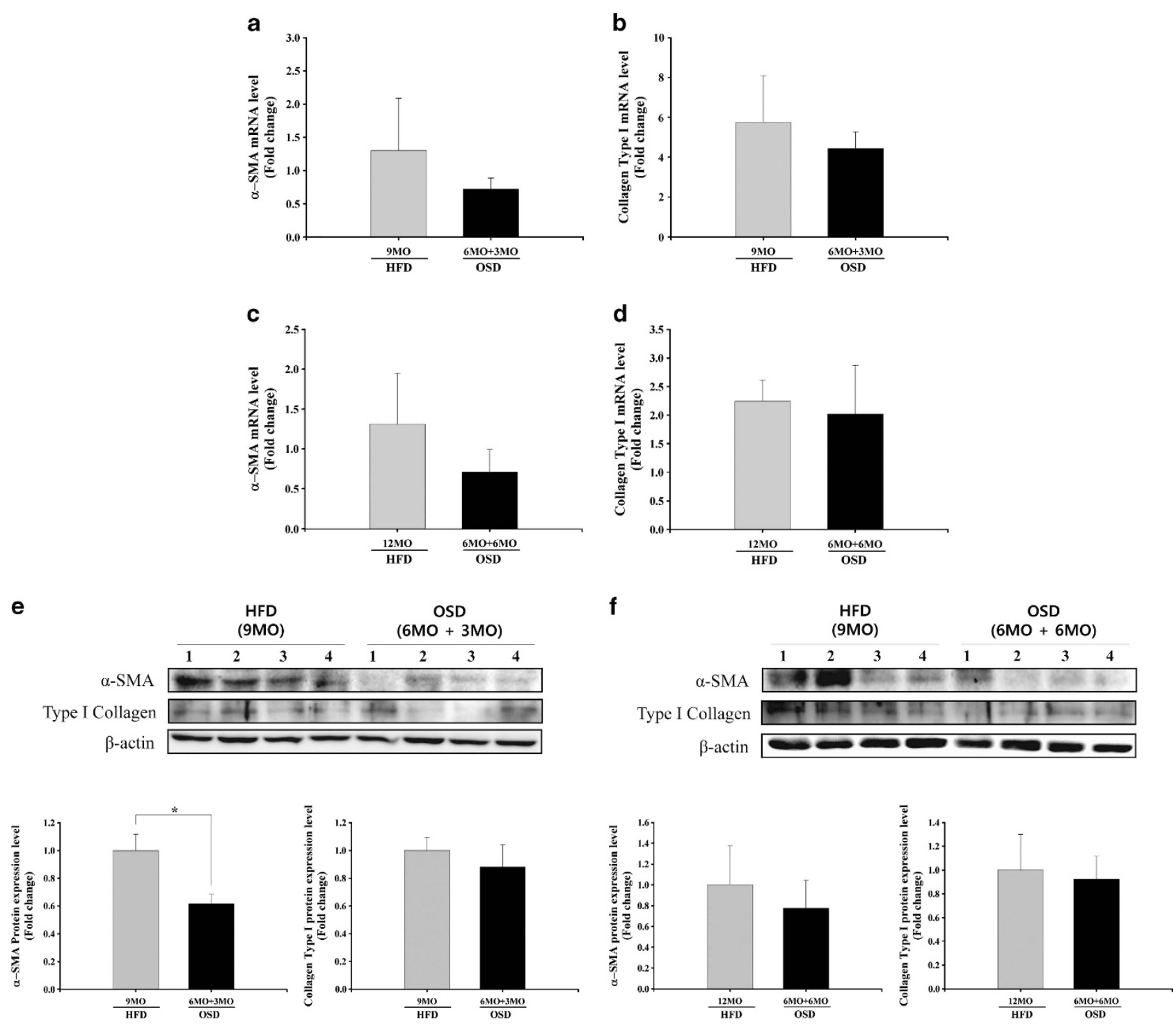

Figure 5 Collagen type I and alpha-smooth muscle actin ( $\alpha$-SMA) expression levels in freshly extracted tissues from the high-fat diet (HFD) and oleuropein-supplemented HFD (OSD) groups after 3 or 6 months of oleuropein treatment in conjunction with the HFD after 6 months of the HFD alone. (a) $\alpha$-SMA mRNA levels in OSD mice after 3 months (means \pm s.e.m.). (b) Collagen type I mRNA levels in OSD mice after 3 months (means \pm s.e.m.). (c) $\alpha$-SMA mRNA levels in OSD mice after 6 months (means \pm s.e.m.). (d) Collagen type I mRNA levels in OSD mice after 6 months (means \pm s.e.m.). (e, f) Collagen type I protein levels and $\alpha$-SMA protein levels in OSD mice after 3 and 6 months (means \pm s.e.m.). HFD vs OSD; $n=4,{ }^{*} P<0.05$.

model animals from the beginning of each study. $12,13,24,25$ Thus, we investigated the therapeutic effects of oleuropein in mice that had already developed NASH to assess the natural HFD-induced progression from NASH to fibrosis. One prior study revealed that the plasma levels of TC, TG and oxidative stress markers, as well as the histopathological features of $\mathrm{NASH}$, were reduced in rats fed $3 \%$ olive leaf extract in oleuropein-supplemented drinking water. ${ }^{24}$ Prior work has also shown that treatment with a diet containing $0.035 \%$ oleuropein for 23 weeks had preventative effects against lipogenesis, high NAS and mild hepatic fibrosis in a spontaneous rat NASH model. ${ }^{25}$ These previous studies suggested the possibility of using oleuropein from olive leaf extract (containing $35 \%$ oleuropein) as a therapy against
NASH. However, these studies did not evaluate the therapeutic effects of purified oleuropein in $\mathrm{NASH}$, which was our aim in this study. Oleuropein has been shown to increase thermogenesis and the secretion of noradrenaline and adrenaline in the brown adipose tissue of rats, ${ }^{12}$ an effect that is important in lipid regulation. In a previous study, we identified protective and therapeutic effects of oleuropein in a NASH OSD mouse model after 3 months of oleuropein treatment. Steatosis in the OSD mouse group was associated with the reduced expression of genes important for lipogenesis, liver fibrosis and inflammatory stress. ${ }^{13}$ These results suggested that oleuropein can prevent hepatic steatosis. However, further investigations were required to evaluate the therapeutic effects of oleuropein on fibrosis. 
To investigate the therapeutic effects of oleuropein on the progression of NASH to fibrosis, we fed NASH mice OSD for an additional 3 or 6 months following an initial 6 months of HFD exposure. The OSD mouse group showed reduced liver damage, morbid obesity and lipid accumulation (as measured by serum biochemical parameters). Furthermore, we confirmed reduced insulin secretion by HOMA-IR in the OSD group. The levels of lobular inflammation and fibrosis were significantly decreased in the OSD group compared with the HFD group. On a molecular level, the expression of $\alpha$-SMA and collagen type I mRNA and protein were significantly reduced in mice fed OSD for 3 and 6 months. The effects of oleuropein were also assessed by histopathological examinations. The fibrosis in the OSD group decreased significantly compared with the HFD group after 3 and 6 months of oleuropein treatment. However, the steatosis grades were not significantly altered in these mice. These results show that oleuropein exhibits only negligible effects on steatosis but that it has a marked impact on fibrosis. This impact is likely the result of irreversible fatty changes in the liver following longterm exposure to HFD. However, in mice fed OSD for 3 and 6 months, NAS scores decreased to levels below those that are indicative of NASH. In addition, the fibrosis grade also improved markedly in the OSD group.

These results show that oleuropein exhibits preventative effects on the progression of NASH to liver fibrosis.

In summary, we reproduced the natural progression of NASH to fibrosis in a HFD-fed mouse model that is characterized by obesity, metabolic abnormalities and histopathological features similar to those observed in human $\mathrm{NASH}$. In this model, oleuropein was shown to exert a preventative effect on the progression of NASH to fibrosis. This effect was first observed in mice with hepatic steatosis by assaying lipid-related biochemical parameters and leptin levels and by assessing histopathological lesions of inflammation and fibrosis in the OSD group. Thus, we suggest that oleuropein may be pharmacologically useful for preventing the progression of steatohepatitis and fibrosis, and it may be a promising therapeutic agent for human NASH.

\section{ACKNOWLEDGEMENTS}

This research was supported by grants from the Korean Health Technology R\&D Project, Ministry of Health \&Welfare (A090282) and the Basic Science Research Program through the National Research Foundation of Korea (NRF), which was funded by the Ministry of Education, Science and Technology (2012-001941).

1 Neuschwander-Tetri BA, Caldwell SH. Nonalcoholic steatohepatitis: summary of an AASLD Single Topic Conference. Hepatology 2003; 37: 1202-1219.

2 Ong JP, Younossi ZM. Epidemiology and natural history of NAFLD and NASH. Clin Liver Dis 2007; 11: 1-16. vii.

3 Farrell GC, Larter CZ. Nonalcoholic fatty liver disease: from steatosis to cirrhosis. Hepatology 2006; 43: S99-S112.
4 Sanyal AJ, Campbell-Sargent C, Mirshahi F, Rizzo WB, Contos MJ, Sterling RK et al. Nonalcoholic steatohepatitis: association of insulin resistance and mitochondrial abnormalities. Gastroenterology 2001; 120: 1183-1192.

5 Brunt EM. Pathology of nonalcoholic steatohepatitis. Hepatol Res 2005; 33: 68-71.

6 Larter CZ, Yeh MM. Animal models of NASH: getting both pathology and metabolic context right. J Gastroenterol Hepatol 2008; 23: 16351648.

7 Anstee QM. Animal models in nonalcoholic steatohepatitis research: utility and clinical translation. Liver Int 2011; 31: 440-442.

8 Omar SH. Oleuropein in olive and its pharmacological effects. Sci Pharm 2010; 78: 133-154.

9 Bravo L. Polyphenols: chemistry, dietary sources, metabolism, and nutritional significance. Nutr Rev 1998; 56: 317-333.

10 Te Sligte K, Bourass I, Sels JP, Driessen A, Stockbrugger RW, Koek GH. Non-alcoholic steatohepatitis: review of a growing medical problem. Eur $J$ Intern Med 2004; 15: 10-21.

11 Cusi K. Role of obesity and lipotoxicity in the development of nonalcoholic steatohepatitis: pathophysiology and clinical implications. Gastroenterology 2012; 142 (711-725), e6.

12 Oi-Kano Y, Kawada T, Watanabe T, Koyama F, Watanabe K, Senbongi R et al. Oleuropein, a phenolic compound in extra virgin olive oil, increases uncoupling protein 1 content in brown adipose tissue and enhances noradrenaline and adrenaline secretions in rats. J Nutr Sci Vitaminol (Tokyo) 2008; 54: 363-370.

13 Park S, Choi Y, Um SJ, Yoon SK, Park T. Oleuropein attenuates hepatic steatosis induced by high-fat diet in mice. J Hepatol 2011; 54: 984-993.

14 Jeen YM, Jin SY. Pathology of nonalcoholic steatohepatitis. Korean J Hepatol 2009; 15: 122-130.

15 Kleiner DE, Brunt EM, Van Natta M, Behling C, Contos MJ, Cummings OW et al. Design and validation of a histological scoring system for nonalcoholic fatty liver disease. Hepatology 2005; 41: 1313-1321.

16 Matthews DR, Hosker JP, Rudenski AS, Naylor BA, Treacher DF, Turner RC. Homeostasis model assessment: insulin resistance and beta-cell function from fasting plasma glucose and insulin concentrations in man. Diabetologia 1985; 28: 412-419.

17 Bedossa P, Houglum K, Trautwein C, Holstege A, Chojkier M. Stimulation of collagen alpha $1(\mathrm{I})$ gene expression is associated with lipid peroxidation in hepatocellular injury: a link to tissue fibrosis? Hepatology 1994; 19: 1262-1271.

18 Anstee QM, Goldin RD. Mouse models in non-alcoholic fatty liver disease and steatohepatitis research. Int J Exp Pathol 2006; 87: 1-16.

19 Brown SD, Peters J. Combining mutagenesis and genomics in the mouseclosing the phenotype gap. Trends Genet 1996; 12: 433-435.

20 Ito M, Suzuki J, Tsujioka S, Sasaki M, Gomori A, Shirakura T et al. Longitudinal analysis of murine steatohepatitis model induced by chronic exposure to high-fat diet. Hepatol Res 2007; 37: 50-57.

21 Xu ZJ, Fan JG, Ding XD, Qiao L, Wang GL. Characterization of high-fat, diet-induced, non-alcoholic steatohepatitis with fibrosis in rats. Dig Dis Sci 2010; 55: 931-940.

22 Chitturi S, Abeygunasekera S, Farrell GC, Holmes-Walker J, Hui JM, Fung $\mathrm{C}$ et al. NASH and insulin resistance: insulin hypersecretion and specific association with the insulin resistance syndrome. Hepatology 2002; 35: 373-379.

23 Uygun A, Kadayifci A, Yesilova Z, Erdil A, Yaman H, Saka M et al. Serum leptin levels in patients with nonalcoholic steatohepatitis. Am J Gastroenterol 2000; 95: 3584-3589.

24 Poudyal H, Campbell F, Brown L. Olive leaf extract attenuates cardiac, hepatic, and metabolic changes in high carbohydrate-, high fat-fed rats. J Nutr 2010; 140: 946-953.

25 Omagari K, Kato S, Tsuneyama K, Hatta H, Sato M, Hamasaki M et al. Olive leaf extract prevents spontaneous occurrence of non-alcoholic steatohepatitis in SHR/NDmcr-cp rats. Pathology 2010; 42: 66-72.

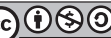

This work is licensed under a Creative Commons Attribution-NonCommercial-ShareAlike 3.0 Unported License. To view a copy of this license, visit http:// creativecommons.org/licenses/by-nc-sa/3.0/ 